\title{
The Difference Of Interpersonal Communication Between College Students Majoring In Art And Science
}

\author{
Lulu Chen ${ }^{1, a}$, Zean Tian ${ }^{2, *}$ \\ ${ }^{1,2}$ School of physics and electronics Hunan University, Changsha 410000, China. \\ a1511451299@qq.com, *tianzean@126.com
}

Keyword: University student, interpersonal communication, Social Network Analysis.

\begin{abstract}
The social network analysis method was used to investigate the interpersonal communication (IC) ability of college students. It is found that art students are outward diffusion, communicate widely and frequently, and the information flow is not easy to be controlled by a few people; while science students are more independent, inward contraction, communicate narrowly and less, and tend to access information via a particular minority. Interpersonal interaction is obviously with art students being slightly stronger. It is useful to improving the university student management model, and helping students make up their IC deficiencies to become comprehensive-developed students, which will lay a solid foundation to integrate in society easier in the future.
\end{abstract}

\section{Introduction}

The interpersonal relationship (IR) is a kind of psychological relationship formed in interacting processes, including the relationship between relatives, friends, alumni/classmates, teacher-student, employee-employer, colleagues/comrades, and leader-subordinates. University stage is critical to improve interpersonal skills. Under the pressure of entrance examination of college/university (hereafter each one will be used at random), the ability to deal with the IR is ignored at primary and secondary stage in our country. Some parents and schools deliberately prevent students from IR, resulting in serious shortage of interpersonal skills and knowledge [1]. After college, most of them will involve directly in the society, face the complex IR, so it is the last chance to enhance and improve the interpersonal skills of students.

The present study investigates the IR difference between students majoring in art and science. It is useful to improve the college student management model, guide students to make up the deficiencies and limitations. It can also help students to adapt society more quickly after graduation and lay the foundation for social integration, so that they can make more contributions for the development of our country.

\section{Research method}

Social network analysis is a set of norms and methods, which analyzes the social network structure and properties [2].In this paper, social network diagram is used to analyze the interpersonal aptitude of university students. Social network graph is a directed graph, in which the points represent individuals in society; connections represent the relation between individuals. Three aspects including the social attributions of the whole network, the centrality, and the small group analysis will be examined in this paper.

\subsection{Social attributions of the whole network}

Basic attributes: density, average distance, cohesion and reciprocity are the standards to measure the relationship between groups of interpersonal relationships.

Density represents a close degree of interpersonal communication in a group, defined as: $d=$ $m /[n(n-1)]$, where $m$ is the number of lines in a chart, $d$ is the density of lines, $n$ is the total number of persons in a community [1]. Average distance is the minimum number of connections between any two points in a network. Cohesion reflects the degree of dispersion and concentration in communication, with value in $[0,1]$. Reciprocity reflects the degree involved with each other in a 
community.

\subsection{Centrality analysis}

Network Centralization (NC): measuring the importance of individuals in a group. A whole class is investigated in our work, and the students with high centrality are the core of the class interaction, while those with low centrality are marginalized in class activities.

$C_{D}\left(n_{i}\right)=d\left(n_{i}\right)=\sum_{j} X_{i j}=\sum_{j} X_{j i}$

$X_{\mathrm{ij}}=0 / 1$, whether the actor $j$ recognizes the relationship with the actor $i$ [3].

$\mathrm{C}_{\mathrm{D}}=\left(\sum_{i=1}^{g}\left[C_{D}\left(n^{*}\right)-C_{D}\left(n_{i}\right)\right]\right) / \max \sum_{i=1}^{g}\left[C_{D}\left(n^{*}\right)-C_{D}\left(n_{i}\right)\right]$

$g$ is the number of persons in a network, $\mathrm{C}_{\mathrm{D}}\left(n^{*}\right)$ is the maximum value of $\mathrm{C}_{\mathrm{D}}\left(n_{i}\right)$ [3].

Intermediary centrality (Network Centralization Index, NCI): representing the extent of controlling the exchanges between others, the ability of a person as a media person. If the intermediary center of a point is 0 , it means that he is not a medium of communication, at the edge of a group or isolated.

$\mathrm{C}_{\mathrm{B}}\left(n_{i}\right)=\left(\sum_{j<k} g_{j k}\left(n_{i}\right)\right) / g_{j k}$

$g_{i k}$ is the shortcut number of actor $j$ to $k, g_{j k}\left(n_{i}\right)$ is the $g_{i k}$ that crosses actor $i$ [3].

Degree centrality: in-degree characterizes the degree of the node to be concerned by other nodes, is an indicator of measuring "opinion leaders", the out-degree illustrates the level of activity of this node in the network.

$$
\mathrm{C}_{D I}\left(n_{i}\right)=d_{I}\left(n_{i}\right)=\sum_{j=1} X_{j i} \quad \mathrm{C}_{D o}\left(n_{i}\right)=d_{o}\left(n_{i}\right)=\sum_{j=1} X_{i j}
$$

\subsection{Small group analysis}

Clique distribution refers to the number of small groups in a group and the member information of each small group, measuring the internal structure of a group.

Clique intensification map is a visualization map of the number of small groups in the group, showing small groups separated from the group and scattered members.

Communication direction of cliques: E-I index=S/G, where S and $G$ are the density of subgroup and group respectively [3]. With E-I 1, -1 , and 0 , the relationship tends to occur outside, inside the community and randomly respectively, indicating the degree of factions (isolated small groups) is greater, smaller and no faction situation.

\section{Data acquisition}

A questionnaire survey was conducted on the art and science students of four classes in a normal college; and the questionnaire data was input into the UCINET software that is preventative in this area, compiled by a group of network analysts at the University of California Irvine. It is now extended by Stephen Borgatti, Martin Everett and Linton Freeman, and the latest version is UCINET v6.415.

\section{Results and Analysis}

\subsection{The overall net social attribution}

Essential attribution: as shown in Fig. 1, the connection between science students is closer than art students. This result is in line with the basic characteristics that art students are outgoing personality, good at expression, and emotion; while the science students are inside collection, rigorous, pragmatic, rational personality, due to long-term learning habits. 

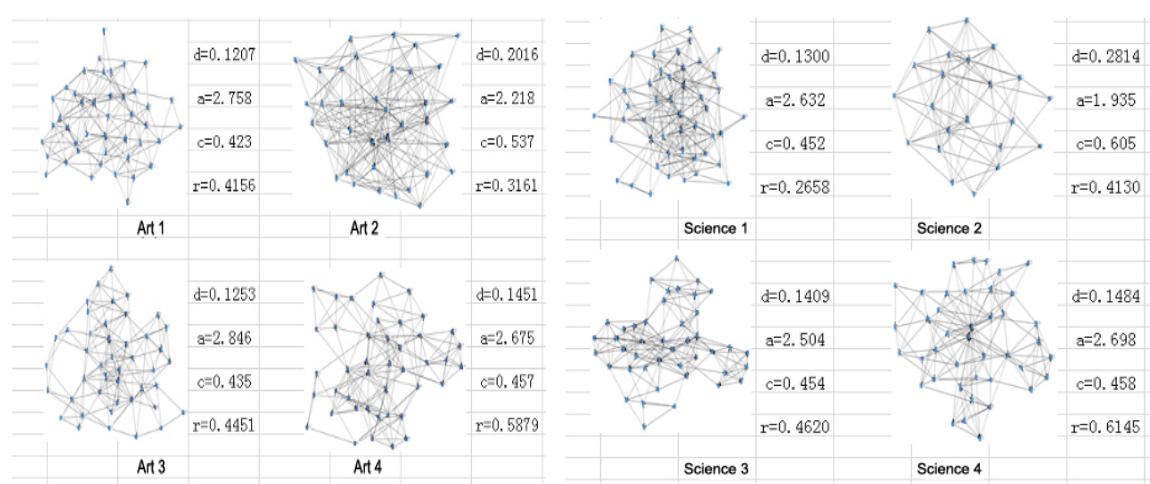

Fig. 1 The overall net social attribution, where $d$ for density, $a$ for average dist ance, $c$ for cohesion, and $r$ for reciprocity.

Social attribution of the whole network: Fig. 1 also shows that the lines are intensive and centralized for art class 2 and science class 1, indicating the members often communicate with each other in the two classes; the attribution for other classes is roughly same, no obvious difference.

\subsection{Central analysis}

Network Centralization (NC): For all classes $\mathrm{NC}_{\max }$ is less than $35 \%$, thus the class cohesion is not high; but with the average of $28.41 \%$, the cohesion of science classes is higher than art ones (23.83\%). This result is consistent with the characteristics of courses that they study. Science needs more collaboration, while art favors autonomous learning [4].

Intermediary centrality: the average NCI of science classes is $14.36 \%$ higher than art classes (9.59\%); thus the members of science classes tend to obtain information from a specific one or a few people. While either value is rather low $(<15 \%)$; thus it is very low that information is monopolized by a few people.

Degree Centralization: the averages of out-degree and of in-degree are respectively $22.05 \%$ and $27.62 \%$ for science classes, slightly higher than art classes (20.92\% and $24.15 \%$ ), so the internal contacts in science classes are tighter.

\subsection{Small group analysis}

Clique distribution: the average of the index of clique distribution shown in Fig. 2 is $52.26 \%$ for art classes larger than the $43.40 \%$ for science classes.

Clique intensification: Fig. 2 also reveals that the clique intensification of art class 3 is the weakest; there are more isolated points and small groups in science class 1; all others are similar.

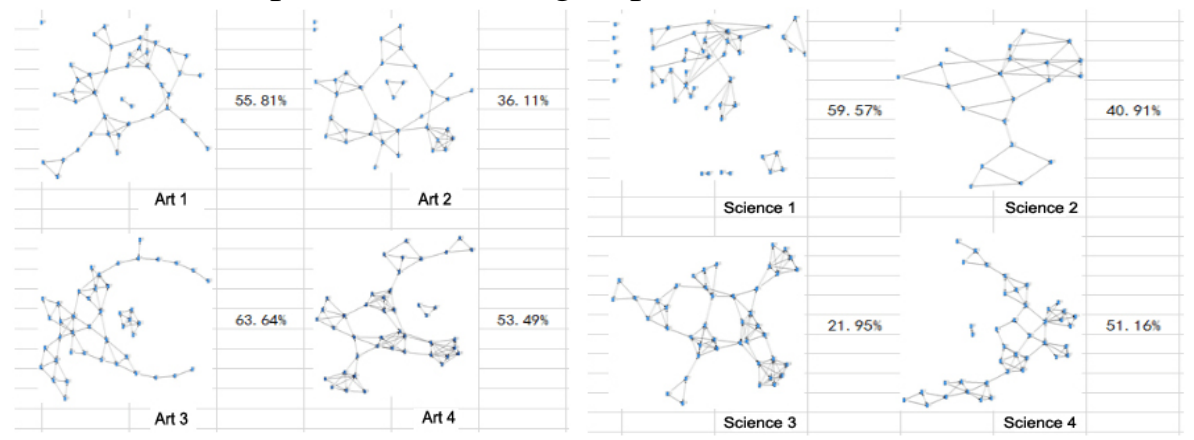

Fig. 2 Clique intensification: the percentages represent the proportion of members outside cliques.

Communication direction of cliques: The average E-I Index for art and science classes has no distinct difference ( 0.494 and 0.441 respectively), thus interaction between members in art and science classes are likely random.

\section{Summary}

The interpersonal communication of college students majoring in art differs from that in science. Art students are wide, spread outward; while science students are narrower, inward contraction. Art students communicate more frequently with each other, information flow is not easy to be 
controlled by a few persons; while science students are more independent, tending to access information via a specific minority. Obvious is the interpersonal group phenomenon for both art and science students, while it is slightly stronger in the school of arts.

\section{Acknowledgments}

This work was supported by the construction of the digital platform for the research of "College Physics"(Grant No. 521201344).

\section{References}

[1] Jiugen Ou-yang, Hui Hua, education management strategy to the interpersonal conflict between college students in dormitory, education and vocational, 2015(24)

[2] Juren Lin, social network analysis: theory, method and application, Beijing normal university press, 2009:41, 93-95.

[3] Jar-Der Luo. Social Network Analysis, M. Social sciences academic press, 2010(2):187-240.

[4] Lan Tian, Yang Liu. Impacting factors of interpersonal communication disorder among normal university students, J. Journal of Hubei Normal University, 2012(2) 